Proceedings

\title{
Tuning of Conditions for Light-Driven Characterization of Poly(phenylacetylene)s ${ }^{\dagger}$
}

\author{
Francisco Rey-Tarrío, Emilio Quiñoá and Félix Freire * \\ Centro Singular de Investigación en Química Biolóxica e Materiais Moleculares (CiQUS) and Departamento \\ de Química Orgánica, Universidade de Santiago de Compostela, E-15782 Santiago de Compostela, Spain; \\ franciscor.tarrio@usc.es (F.R.-T.); \\ * Correspondence: felix.freire@usc.es.com \\ + Presented at the 24th International Electronic Conference on Synthetic Organic Chemistry, 15 November- \\ 15 December 2020; Available online: https://ecsoc-24.sciforum.net/.
}

Received: date;

\begin{abstract}
Poly(phenylacetylene)s are a family of dynamic helical polymers where the elongation and the helical sense of the double helix can be easily tuned in a controlled way using the proper external stimuli. Since there is a direct relationship between the secondary structure of the polymer and its potential applications, the structural elucidation of these polymers is nowadays a challenge in polymer chemistry. These polymers are characterised using the combination of different techniques such as CD, UV-Vis, Raman, DSC... However, the interpretation of the data is time consuming and in occasions difficult, being necessary to resort to $\mathrm{AFM}^{4}$. Due these obstacles, we focus on the light-driven cyclization of PPAs as a fast and easy-going alternative approach to decipher the configuration of the polyene backbone. Several parameters must be considered to achieve acceptable values of reproducibility and repeatability on this technique. On this work, we evaluate the influence of the relation between volume/concentration of sample and photoabsorption on PPAs when monitoring results by UV-Vis, resulting in and easy and controlled way to tune the light-driven characterization of PPAs.
\end{abstract}

Keywords: photochemistry; poly(phenylacetylene)s; helical structure; cis-cisoid; cis-transoid

\section{Introduction}

In natural proteins, the secondary structure is strongly related to their functions. Based on this concept, many synthetic polymers have been prepared in the last decades with the propose of creating new materials with applications as sensors, data storage, etc. [1]. However, the determination of the secondary structure, responsible of these applications, has only been demonstrated in some cases.

Poly(phenylacetylene)s are one of these new materials, with interesting properties as sensors and chiral recognition. These polymers can adopt four structures in their formation. However, only two of these promote the formation of a helical structure: the cis-cisoid $(c-c)$ conformation, more compressed, and the cis-transoid ( $c-t)$ conformation, more stretched.

The characterization of the helix is based on the combination of different techniques as DSC [2], TGA, Raman, CD [3] and UV-Vis that provide information to determinate if a polymer is $c-c$ or $c-t$. Nevertheless, these techniques have larger limitations, resulting in many cases in ambiguous results.

For the fully and unequivocal determination of the secondary structure, it is required the combination of AFM and theorical calculations [4]. These techniques provide information about the structural parameters of the helix: residues per turn, dihedral angle, helical pitch and package angles [5]. This information allows us to know, with unparalleled precision, the helical conformation of the 
polymer. Despite their great advantages, the scope of this methodology is very limited for the success in the preparation of a good 2D crystal.

Due to the limitations in the current methodologies for characterization of helical conformations on PPAs, being helical polymers currently one of the hottest topics in polymer chemistry, we focused in solve this problem. For this reason, we develop a new methodology that provides an easy and fast method to determine the helical conformation of the polymer based on a photochemistry unfolding of the helix, finding the answer to one of the big current challenges in polymer chemistry.

As part of this challenge, under visible light irradiation we must control several parameters to achieve reproducibility results for a fully and unequivocal characterization of PPAs. Thus, we found that the volume of the sample is an essential factor to get homogeneous values and even an easy way to modulate at will the photoreaction rates.

\section{Results and Discussion}

From previous works, it is known that the $(R)-\alpha$-methoxy- $\alpha$-trifluoromethylphenylacetic acid (MTPA) pendant group presents a cis-trans equilibrium around the $(\mathrm{H}-) \mathrm{N}-\mathrm{C}(=\mathrm{O})$ amide bond. This equilibrium can be modified with external stimuli, promoting in the polymer (poly-(R)-1) a preferred conformation: cis-transoid (cis amide) or cis-cisoid (trans amide) [6].

In non-donor/non-polar solvents, as $\mathrm{CHCl}_{3}$, poly-( $\left.R\right)-\mathbf{1}$ was fully characterized. The combination of different techniques, specially AFM images and theorical calculations, show that in solid phase adopts a right-handed backbone in a compressed helical structure with three residues per turn, $3 \mathrm{~nm}$ of helical pitch and $50^{\circ}$ of packing angles. These parameters correspond to a cis-cisoid $\left(+70^{\circ}\right)$ helical structure (Figure 1a). Thus, we evaluated the differences of this well-known structure under irradiation with visible light for different sample volumes.

To develop these experiments, a solution of poly- $(R)-\mathbf{1}$ in chloroform $\left(9.00 \times 10^{-4} \mathrm{M}\right)$ was prepared. Separately, 1, 7 and $14 \mathrm{~mL}$ of solution was purged under argon atmosphere and irradiated under visible light. Monitoring CD and UV-Vis, we determine the disappearance of the backbone absorption for each solution (e.g., $7 \mathrm{~mL}$, Figure 1b).

The results (Figure 1c) were represented as a normalized UV absorption at a fixed wavelength $(390 \mathrm{~nm})$ versus time of irradiation. Thus, even though a constant increase of irradiation time was expected with the increasing amount of sample, normalized UV vs. time of irradiation explains two types of behavior for these three samples.

Differences between 1 and 7/14 mL cannot be assumed only as result of volume of samples based on $7 / 14 \mathrm{~mL}$ comparison. Thus, a second effect influences the $1 \mathrm{~mL}$ sample. As the reaction is exothermic, we assumed that decreasing the volume of sample the same number of photons are absorbed in a smaller space, causing a higher temperature rise. As probe of concept, $7 \mathrm{~mL}$ of solution was analyzed again at 50 degrees resulting in a Normalized UV $=0$ for $t=30 \mathrm{~min}(57 \%$ lower than a standard temperature, 20 degrees). 


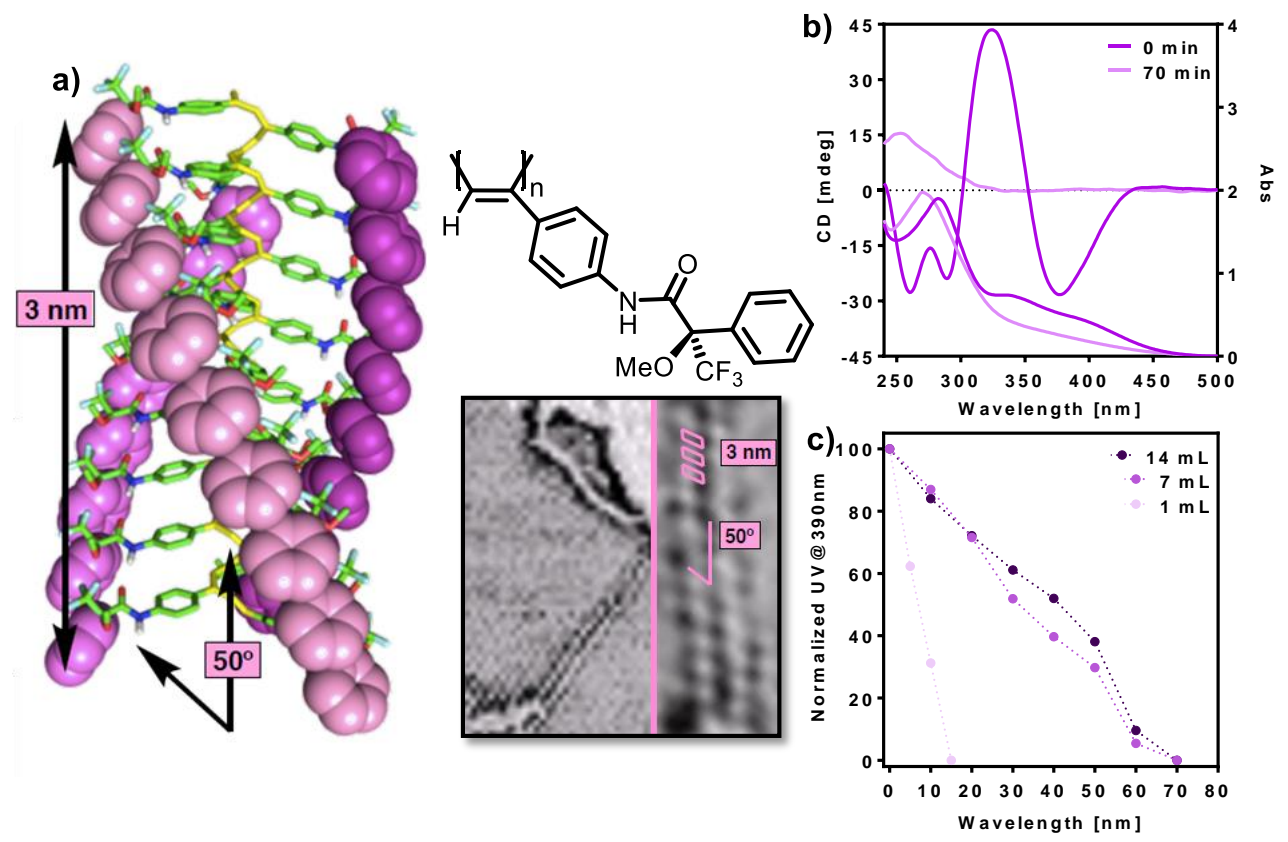

Figure 1. (a) 3D structures and AFM image of poly-( $R$ )-1 in non-donor solvent $\left(\mathrm{CHCl}_{3}\right.$, cis-cisoidal) (b) ECD and UV-vis spectra of for poly-(R)-1 before and after irradiation with visible light. (c) Normalized UV signal decay vs. time for poly- $(R)-\mathbf{1}$ at different times under irradiation with visible light for different volumes of sample.

\section{Conclusions}

In conclusion, it has been shown that the volume of sample can generate a catalytic effect. Thus, we propose that the influence of this effect is conditioned by the increase in temperature by reducing the space available to absorb the incident radiation. A first analysis shows a similar effect by increasing the temperature of the sample. However, as temperature influences not only the reaction rate, but also the stability of the polyene, further studies are being developed to better understand the variables that allow us to modulate the photoreactivity of poly(phenylacetylene)s.

\section{Materials and Methods}

$\mathrm{CD}$ measurements were done in a Jasco-720. The amount of polymer used for $\mathrm{CD}$ measurements were $0.3 \mathrm{mg} / \mathrm{mL}$ in $\mathrm{CHCl}_{3}$.

UV spectra were registered in a Jasco V-630. The amount of polymer used for UV measurements were $0.3 \mathrm{mg} / \mathrm{mL}$ in $\mathrm{CHCl}_{3}$.

Irradiation experiments were done in an Asahi Spectra Xenon light model MAX-303. The amount of polymer use for irradiations were $0.3 \mathrm{mg} / \mathrm{mL}$ using visible light $(\lambda=350-550 \mathrm{~nm})$.

Acknowledgments: Financial support from MINECO (PID2019-109733GB-I00), Xunta de Galicia (ED431C 2018/30, Centro Singular de Investigación de Galicia acreditación 2019-2022, ED431G 2019/03 and F. R. T. for predoctoral fellowships) and the European Regional Development Fund (ERDF) is gratefully acknowledged.

Conflicts of Interest: The authors declare no conflict of interest.

\section{References}

1. Yashima, E.; Ousaka, N.; Taura, D.; Shimomura, K.; Ikai, T.; Maeda, K. Supramolecular Helical Systems: Helical Assemblies of Small Molecules, Foldamers, and Polymers with Chiral Amplification and Their Functions. Chem. Rev. 2016, 116, 13752-13990.

2. Liu, L.; Namikoshi, T.; Zang, Y.; Aoki, T.; Hadano, S.; Abe, Y.; Wasuzu, I.; Tsutsuba, T.; Teraguchi, M.; Kaneko, T. Top-Down Preparation of Self-Supporting Supramolecular Polymeric Membranes Using 
Highly Selective Photocyclic Aromatization of Cis-Cisoid Helical Poly(phenylacetylene)s in the Membrane State. J. Am. Chem. Soc. 2013, 135, 602-605.

3. Nieto-Ortega, B.; Rodríguez, R.; Medina, S.; Quiñoá, E.; Riguera, R.; Casado, J.; Freire, F.; Ramírez, F.J. Sequential Induction of Chirality in Helical Polymers: From the Stereocenter to the Achiral Solvent. J. Phys. Chem. Lett. 2018, 9, 2266-2270.

4. Yoshida, Y.; Matawari, Y.; Motoshige, A.; Motoshige, R.; Hiraoki, T.; Wagner, M.; Müllen, K.; Tabata, M. Accordion-like Oscillation of Contracted and Stretched Helices of Polyacetylenes Synchronized with Restricted Rotation of Side Chains. J. Am. Chem. Soc. 2013, 135, 4110-4116.

5. Fernández, B.; Rodríguez, R.; Rizzo, A.; Quiñoá, E.; Riguera, R.; Freire, F. Predicting the Helical Sense of Poly(phenylacetylene)s from their Electron Circular Dichroism Spectra. Angew. Chem. Int. Ed. 2018, 57, 3666-3670.

6. Leiras, S.; Freire, F.; Seco, J.M.; Quiñoá, E.; Riguera, R. Controlled modulation of the helical sense and the elongation of poly(phenylacetylene)s by polar and donor effects. Chem. Sci. 2013, 4, 2735-2743.

Publisher's Note: MDPI stays neutral with regard to jurisdictional claims in published maps and institutional affiliations.

(C) 2020 by the authors. Submitted for possible open access publication under the terms and conditions of the Creative Commons Attribution (CC BY) license (http://creativecommons.org/licenses/by/4.0/). 